\title{
Hysteresis, Switching and Anomalous Behaviour of a Quartz Tuning Fork in Superfluid ${ }^{4} \mathrm{He}$
}

\author{
D.I. Bradley • M.J. Fear • S.N. Fisher • A.M. Guénault • R.P. Haley • \\ C.R. Lawson • G.R. Pickett • R. Schanen • V. Tsepelin • L.A. Wheatland
}

Received: 25 July 2013 / Accepted: 19 September 2013 / Published online: 3 October 2013

(C) The Author(s) 2013. This article is published with open access at Springerlink.com

\begin{abstract}
We have been studying the behaviour of commercial quartz tuning forks immersed in superfluid ${ }^{4} \mathrm{He}$ and driven at resonance. For one of the forks we have observed hysteresis and switching between linear and non-linear damping regimes at temperatures below $10 \mathrm{mK}$. We associate linear damping with pure potential flow around the prongs of the fork, and non-linear damping with the production of vortex lines in a turbulent regime. At appropriate prong velocities, we have observed metastability of both the linear and the turbulent flow states, and a region of intermittency where the flow switched back and forth between each state. For the same fork, we have also observed anomalous behaviour in the linear regime, with large excursions in both damping, resonant frequency, and the tip velocity as a function of driving force.
\end{abstract}

Keywords Superfluid He-4 · Turbulence · Critical velocity · Tuning fork

\section{1 introduction}

Previous experiments in superfluid ${ }^{4} \mathrm{He}$ have demonstrated the creation of quantum turbulence [1] when the velocity of oscillating quartz tuning forks [2] exceeds a critical value [3-6]. In a typical experiment the driving force on the resonant tuning fork is gradually increased in small steps, and the peak velocity is measured. At low velocities the flow of helium around the fork is laminar and the velocity is accurately proportional to the driving force. When the velocity of the fork oscillations exceeds a critical value, then some additional damping is observed arising from the generation of quantum turbulence. Two regimes of oscillations can be clearly distinguished, the

D.I. Bradley · M.J. Fear · S.N. Fisher · A.M. Guénault · R.P. Haley · C.R. Lawson ( $)$ •

G.R. Pickett · R. Schanen · V. Tsepelin · L.A. Wheatland

Department of Physics, Lancaster University, Lancaster, UK

e-mail: c.lawson@lancaster.ac.uk 
Fig. 1 Typical hysteretic behaviour observed during the transition from the laminar to the turbulent regime and then back to the laminar regime. The different colors shows different cycles that accurately superimpose, demonstrating the reproducibility of the force-velocity curves away from the transition region and the good stability of the signal. In the transition region the fork response exhibits strongly hysteric behaviour. These data were taken at $7 \mathrm{mK}$ and $1 \mathrm{bar}$ (Color figure online)

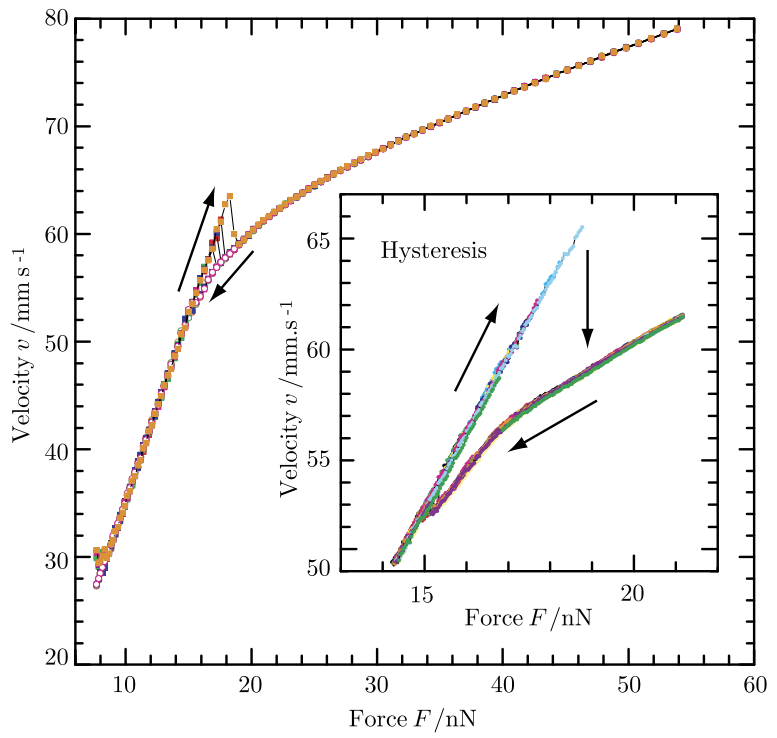

linear laminar regime at low velocities and the turbulent regime above the critical velocity. Furthermore, in some experiments it has also been observed that hysteresis is present in the critical velocity at which switching occurs between these two regimes, not only for forks [3] but also for vibrating wires [7, 8] and oscillating spheres [9-12]. Away from the critical velocity region, the force-velocity relationship is predictable and reversible, but close to the change of regime the behaviour is much more interesting. We find that both the laminar and turbulent regime can exist in meta-stable states as shown in Fig. 1. Here in Sect. 3 we investigate the switching between these states, and in Sect. 4 we report some anomalous damping effects observed in the cold superfluid.

\section{Experimental Cell}

The experimental cell is shown in Fig. 2. The cell has a thick-walled cylinder made of epoxy resin and is designed to withstand pressures up to 34 bar. The ${ }^{4} \mathrm{He}$ sample is cooled using silver-sintered heat exchangers welded to a silver wire which is connected to sinters in the mixing chamber of a dilution refrigerator. A heater on the wire and a thin film $\mathrm{RuO}_{2}$ thermometer resistor inside the cell allows temperature measurement and regulation of the helium.

The piezoelectric fork is set into motion by applying an AC voltage near the resonant frequency, using a waveform generator and suitable attenuators. The motion of the fork prongs generates an electric current, proportional to their velocity, that is detected using a custom-made current-to-voltage converter [13]. This voltage is fed to a lock-in amplifier phase-locked to the waveform generator. We can therefore measure the in-phase and out-of-phase velocity response of the fork to the driving force. 


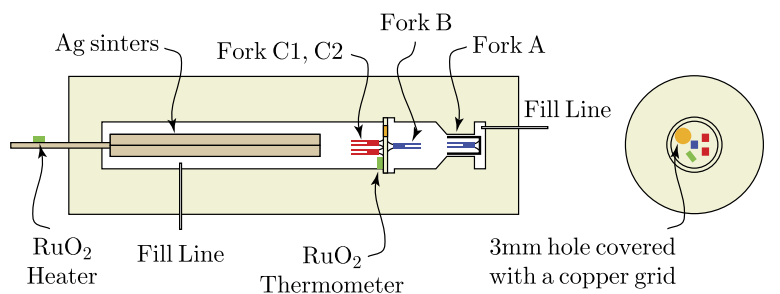

Fig. 2 The experimental cell contains 4 identical forks (part label KDS5M from KDS America/Daishinku Corporation). All the measurements reported here were made on fork A, which was kept in its original cylindrical container with the end cap removed. Two $\mathrm{RuO}_{2}$ resistors are used one as a thermometer and one a heater. Most of the volume is occupied by silver sinter heat exchangers used to cool the ${ }^{4} \mathrm{He}$ sample (Color figure online)

In these measurements a continuous tracking routine is used to keep fork A on resonance. The routine adjusts the driving frequency until the out-of-phase signal (which is zero at resonance) becomes less than $1 \%$ of the in-phase signal. Thus, while tracking the resonant frequency, we can observe the velocity response as a function of driving force, as in Fig. 1. Alternatively we can simply monitor the fork velocity at a fixed driving voltage as a function of time, looking for transitions between the turbulent and the laminar state, as discussed below.

The electrical data (applied voltage $V$ and current response $I$ ) are converted to mechanical data (applied force and velocity response) using the fork constant $a$. This is determined from conventional frequency sweeps (to give a value of "height times width over drive" of the resonance) and from previous direct prong velocity measurements [14]. We find the value of $a$ for our miniature forks to be $2.1 \times 10^{-6} \mu \mathrm{C} / \mathrm{m}$. Hence we determine the applied force $F=a V / 2$ and the velocity $v=I / a$.

\section{Hysteretic Behaviour}

In Fig. 3 we give the velocity behaviour from many identical cycles of the tuning fork, as the driving force is increased and then decreased across the transition region. All these cycles were made at $1 \mathrm{bar}, 7 \mathrm{mK}$ and over the same time period.

The laminar state is found to be stable for velocities below $53.4 \mathrm{~mm} \mathrm{~s}^{-1}$. Above this value, the probability that the fork motion falls to the turbulent state increases with the velocity. Conversely, the turbulent state is seen to be stable above $53.4 \mathrm{~mm} \mathrm{~s}^{-1}$, below which the probability that the fork motion jumps back into the laminar state increases with decreasing velocity. The lower insert in Fig. 3 shows the number of events corresponding to a transition as a function of the driving force. It shows that during one cycle, the likeliness of a turbulent-to-laminar transition finds its maximum at about $52.6 \mathrm{~mm} \mathrm{~s}^{-1}$ while the likeliness of a laminar-to-turbulent event peaks at about $61 \mathrm{~mm} \mathrm{~s}^{-1}$, but is somewhat more spread out.

Next, it can be seen the velocity $53.4 \mathrm{~mm} \mathrm{~s}^{-1}$ defines an overlapping region of driving force, within which the fork can intermittently switch between laminar and turbulent states. The top insert shows an example of this spontaneous switching. Analysis of this switching, using methods analogous to those of Schoepe et al. [11, 12] forms part of a separate study [15]. 


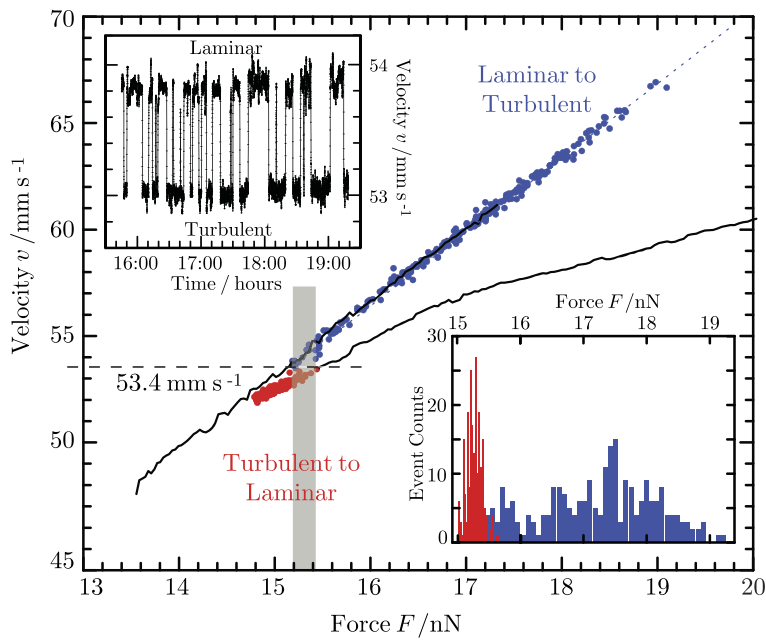

Fig. 3 A series of force-velocity curves is shown, during which we observed jumps between the pure potential flow (laminar) state and the turbulent state. The data points represent the instant at which the fork suddenly jumps from one state to the other (blue for jumps down from the laminar state, red for up from the turbulent state). The frequency of both sets of jumps is plotted in the bottom right insert. In the intermediate region (shaded grey) the fork spontaneously jumps back and forth between the two states as shown in the top left insert, data taken when monitoring the fork velocity over time at a fixed driving force. The meta-stable regions are bounded by a characteristic velocity of $53.4 \mathrm{~mm} \mathrm{~s}^{-1}$ (Color figure online)

Fig. 4 Frequency sweep results showing anomalous damping. The measured damping width and frequency from a series of frequency sweeps is shown, while the sample is kept at $5 \mathrm{mK}$ and $10 \mathrm{bar}$. The frequency is seen first to increase a little as the width increases dramatically. Then as the maximum damping width is approached, the frequency falls rapidly and the width starts to decrease back towards its normal low value. After the event, the width again is very low, but the frequency is significantly lower than the initial value (Color figure online)

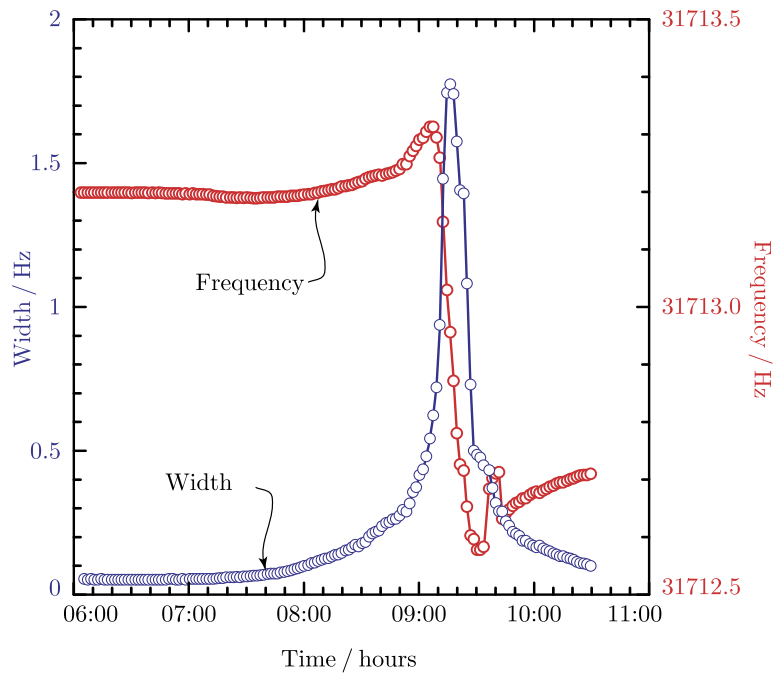

\section{Anomalous Damping}

On several rare occasions when measuring fork $\mathrm{A}$ in an earlier cell, we observed unexpected and mysterious damping effects, some of which appear similar to effects reported by others $[6,16]$ that were explained by coupling to resonant sound modes in the surrounding cell. We observed these effects in both frequency sweeps (see Fig. 4) 
Fig. 5 Force-velocity curves showing anomalous damping. Note the much wider range of these curves than those of Fig. 1 and Fig. 3. The upper (black) curve shows the usual situation. The other curves, starting from the lowest (purple) curve give a time sequence of the recovery towards normality from anomalous damping. Note that the additional damping occurs in the laminar and intermediate regions, but all measurements collapse to the same turbulent curve at high drives. These measurements were made at around $50 \mathrm{mK}$ and 10 bar (Color figure online)

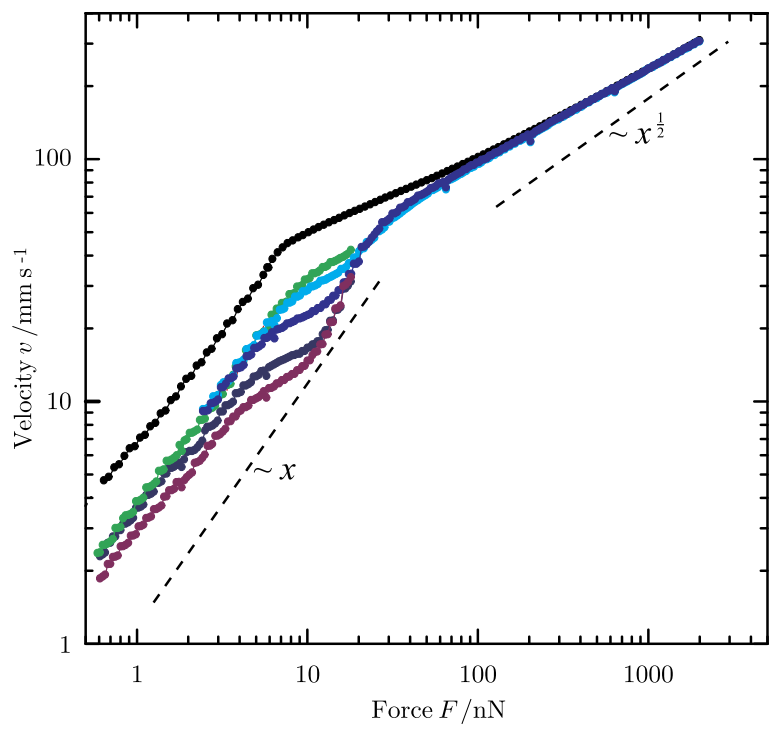

and force-velocity cycles (see Fig. 5). The occurrence of these events appeared to be spontaneous. In the case depicted in Fig. 4, we had left the fork doing regular frequency sweeps during the night. The extra damping process started early in the morning after an event-less night of about 10 hours, and there was no indication of what could have triggered it. The figure shows the measured centre frequency and frequency width of each sweep. The overall event took a relatively long time, occurring over a period of 3 hours. Attempts to trigger the process actively, using mechanical sources of noise or unusually large drive values were unsuccessful. However, we did once manage to kill the process using a small deliberate pressure pulse, which reset the fork to the usual narrow resonance curve. Once, when we found the fork in the anomalous damping condition, we performed a series of force-velocity sweeps whilst the effect was "wearing off", as shown in Fig. 5. In these cases, the damping in the laminar region was greatly increased, initially by a factor of about 4 , which gradually reduced. In the transition region the anomalous curves have a surprising sigmoid shape, and seem to join together before finally meeting the usual turbulent curve.

\section{Conclusion}

We have studied the hysteretic transition to and from laminar and turbulent states of a tuning fork in superfluid ${ }^{4} \mathrm{He}$ at millikelvin temperatures. There is rich preliminary data in Fig. 3 both from driving force sweeps, and from spontaneous transitions. Work is in progress to analyse the statistics of the switching process.

During these studies, at temperatures low enough to eliminate the likelihood of crossing second sound resonances, we have observed some surprising occasional changes in damping, which appears and then anneals out. We can not rule out the possibility that some of the anomalies are due to acoustic emission of first sound that 
couples resonantly to modes in our cell. However, for some of the events we have seen, it seems that the increased damping is associated with a frequency shift downward, possibly indicating a reduction in stiffness experienced by the fork. This leads us to speculate that some of the anomalous behaviour may be due persistent vortex lines are trapped on our fork, either between the prongs or between the fork and its container, which increase the restoring force; but that they eventually find a way to disperse during which time Kelvin waves produce dissipation.

Acknowledgements We thank S.M. Holt, A. Stokes and M.G. Ward for excellent technical support. This research is supported by the UK EPSRC and by the European FP7 Programme MICROKELVIN Project, no. 228464.

Open Access This article is distributed under the terms of the Creative Commons Attribution License which permits any use, distribution, and reproduction in any medium, provided the original author(s) and the source are credited.

\section{References}

1. W.F. Vinen, J.J. Niemela, J. Low Temp. Phys. 128, 167 (2002)

2. R. Blaauwgeers, M. Blažková, M. C̆lovec̆ko, V.B. Eltsov, R. de Graaf, J. Hosio, M. Krusius, D. Schmoranzer, W. Schoepe, L. Skrbek, P. Skyba, R.E. Solntsev, D.E. Zmeev, J. Low Temp. Phys. 146, 537 (2007)

3. D.I. Bradley, M.J. Fear, S.N. Fisher, A.M. Guénault, R.P. Haley, C.R. Lawson, P.V.E. McClintock, G.R. Pickett, R. Schanen, V. Tsepelin, L.A. Wheatland, J. Low Temp. Phys. 156, 116 (2009)

4. G.A. Sheshin, A.A. Zadorozhko, E.Y. Rudavskii, V.K. Chagovets, L. Skrbek, M. Blažková, Low Temp. Phys. 34, 875 (2008)

5. M. Blažková, D. Schmoranzer, L. Skrbek, W.F. Vinen, Phys. Rev. B 79, 054522 (2009)

6. D. Garg, V.B. Efimov, M. Giltrow, P.V.E. McClintock, L. Skrbek, W.F. Vinen, Phys. Rev. B 85, 144518 (2012)

7. D.I. Bradley, D.O. Clubb, S.N. Fisher, A.M. Guénault, R.P. Haley, C.J. Matthews, G.R. Pickett, K.L. Zaki, J. Low Temp. Phys. 138, 493 (2005)

8. N. Hashimoto, A. Handa, M. Nakagawa, K. Obara, H. Yano, O. Ishikawa, T. Hata, J. Low Temp. Phys. 148, 299 (2007)

9. J. Jäger, B. Schuderer, W. Schoepe, Phys. Rev. Lett. 74, 566 (1995)

10. M. Niemetz, H. Kerscher, W. Schoepe, J. Low Temp. Phys. 126, 287 (2002)

11. W. Schoepe, Phys. Rev. Lett. 92, 095301 (2004)

12. M. Niemetz, W. Schoepe, J. Low Temp. Phys. 135, 447 (2004)

13. S. Holt, P. Skyba, Rev. Sci. Instrum. 83(6), 064703 (2012)

14. D.I. Bradley, P. Crookston, M.J. Fear, S.N. Fisher, G. Foulds, D. Garg, A.M. Guénault, E. Guise, R.P. Haley, O. Kolosov, G.R. Pickett, R. Schanen, V. Tsepelin, J. Low Temp. Phys. 161, 116 (2010)

15. To be published

16. D. Schmoranzer, M. La Mantia, G. Sheshin, I. Gritsenko, A. Zadorozhko, M. Rotter, L. Skrbek, J. Low Temp. Phys. 163, 317 (2011) 